\title{
Stromal CD10 expression in invasive breast carcinoma correlates with poor prognosis, estrogen receptor negativity, and high grade
}

\author{
Nikita A Makretsov ${ }^{1,3}$, Malcolm Hayes ${ }^{2}$, Beverley A Carter ${ }^{3}$, Shahriar Dabiri ${ }^{1}$, C Blake Gilks ${ }^{1}$ \\ and David G Huntsman ${ }^{1}$ \\ ${ }^{1}$ Genetic Pathology Evaluation Centre of the Department of Pathology and Prostate Research Centre of \\ Vancouver General Hospital, British Columbia Cancer Agency and University of British Columbia, \\ Vancouver, BC, Canada; ${ }^{2}$ Department of Pathology, British Columbia Cancer Agency, Vancouver, BC, Canada \\ and ${ }^{3}$ Department of Pathology and Laboratory Medicine, Memorial University of Newfoundland, \\ St John's, NL, Canada
}

\begin{abstract}
CD10 is a zinc-dependent peptidase (metalloproteinase), which degrades a variety of bioactive peptides. Earlier studies suggested that CD10 expression in tumor stroma is associated with biological aggressiveness of the tumor. To date, only one study has addressed the clinical significance of stromal CD10 expression in invasive carcinoma of the breast. The aim of this confirmatory study is to evaluate stromal CD10 expression in breast carcinoma and to examine associations between CD10, clinicopathological variables, and patient outcome. Tissue microarrays, containing $\mathbf{4 3 8}$ cases of invasive breast carcinoma and $\mathbf{1 5}$ cases of ductal carcinoma in situ with 15 years median follow-up time, were assembled. CD10 expression was assessed by immunohistochemistry and scored as negative, weak and strong. Nonparametric correlational tests, univariate and multivariate survival analyses were performed. Stromal CD10 was preferentially expressed in invasive compared to noninvasive breast cancers $(P=0.003)$. There were correlations between stromal CD10 expression and higher tumor grade $(P=0.01)$ and estrogen receptor $(E R)$ negative status $(P=0.002)$. There was no correlation between CD10 and lymph node status, tumor size, histological subtype, progesterone receptors, and Her2 status. Stromal CD 10 expression was associated with decreased long-term disease-specific and overall survival in the entire cohort $(P<0.01)$, and in lymph node negative $(P<0.05)$, but not lymph node positive subset of patients. It approached prognostic significance in multivariate analysis $(P=0.06)$ when lymph node status, tumor size, ER and Her2 were considered in the same model; and was associated with a relative risk of death of 2.8, compared to relative risk of 2.4 for lymph node positive status. Thus, stromal CD10 expression in invasive carcinoma of the breast is associated with ER negativity, higher tumor grade and decreased survival and constitutes a potential prognostic marker and a target for development of novel therapies.
\end{abstract}

Modern Pathology (2007) 20, 84-89. doi:10.1038/modpathol.3800713; published online 24 November 2006

Keywords: CD10; breast; stroma; invasive carcinoma; prognosis

The zinc-dependent peptidase (metalloproteinase)-CD10, is commonly expressed in bone marrow lymphoid stem cells, pro-B lymphoblasts, mature neutrophils, various lymphoma subtypes, renal cell carcinoma and endometrial stromal sarcoma cells. CD10-positive cells have been reported in the stroma of prostate, ${ }^{1}$ breast, ${ }^{2,3}$ color-

Correspondence: Dr NA Makretsov, MD, PhD, Department of Pathology and Laboratory Medicine, Health Care Corporation of St John's, 300 Prince Philip Drive, St John's, NL, Canada A1B3V8. E-mail: nmakrets@yahoo.com

Received 22 February 2006; revised 9 May 2006; accepted 9 July 2006; published online 24 November 2006 ectal, ${ }^{4}$ and lung carcinomas. ${ }^{5,6}$ CD10 positivity has also been reported in stromal myoepithelial cells from normal breast tissue and benign myoepithelial tumors. ${ }^{3,7-9}$ Several reports indicated that stromal CD10 expression is associated with biological aggressiveness in various epithelial malignancies. ${ }^{2,5,10,11}$ In gastric carcinoma CD10-positive stroma correlates with vascular invasion and metastasis. ${ }^{10}$ Stromal CD10 is expressed with a higher frequency in malignant, as opposed to borderline and benign phyllodes tumors. ${ }^{11}$ To date, only one study has examined the expression of CD10 in stromal cells around the infiltrating tumor cells in invasive breast carcinomas and showed its association with a worse prognosis. ${ }^{2}$ 
The aims of this study were: (1) to estimate the frequency of expression of stromal CD10 expression in invasive breast carcinomas and ductal carcinomas in situ (DCIS); (2) to assess prognostic significance of stromal CD10 expression in univariate and multivariate analysis using long-term follow-up data (more than 15 years); (3) to examine the association of stromal CD10 expression with the clinicopathological parameters.

\section{Materials and methods}

\section{Tissue Microarrays, Grading, Immunostaining Scoring and Data Processing}

Tissue microarrays containing 438 cases of invasive breast carcinoma and 15 cases of DCIS (Vancouver General Hospital, from 1974 to 1995) were assembled, as described earlier. ${ }^{10}$ A total four $0.6 \mathrm{~mm}$ tissue cores per case were taken. The tumor cores were extracted from representative areas of tumor from paraffin embedded, formalin-fixed tumor tissue blocks, according to corresponding H\&E stained whole section slides. There was no attempt to select areas of tumor with specific growth patterns or stromal predominance. The H\&E slides and scanned images were graded according to modified Scarth-Bloom-Richardson grading system for invasive breast carcinoma and Consensus Conference on the classification of ductal carcinoma in situ. ${ }^{12}$

CD10 expression in the tumor stroma (both in stromal cells and extracellular matrix) was assessed by immunohistochemistry by standard immunoperoxidase method using an automatic DAKO immunostainer (monoclonal CD10, Novocastra, dilution 1:50, pretreatment with Decloak $5 \mathrm{~min}$ in TRS). The staining was scored semiquantitatively as negative, weak (either diffuse weak staining or weak or strong focal staining in less that $30 \%$ of stromal cells per core) and strong (defined as strong staining of $30 \%$ or more of the stromal cells). The tissue microarray slides were scored independently by two pathologists, the discrepancies were resolved by consensus. Overall agreement of scoring data was measured by $\kappa$-statistics. In case of variable results between individual tumor cores taken from the same tumor, the higher tumor score was considered a final score and superseded all other scores. Only cases with all four cores containing tumor cells were considered for statistical analysis in order to address possible heterogeneity of the staining in various tumor portions. Tissue cores with stromal tissue only, but without representative tumor cells were excluded from analysis. The score data were entered into electronic spread-sheets (Exel), and were processed using Deconvoluter software, as described previously. ${ }^{13}$ SPSS 11.0 (Chicago, IL, USA) statistical package was used for data analysis.

\section{Statistical Analysis}

For each patient, the date of breast cancer diagnosis, date of last follow-up, vital status at last follow-up, causes of death (breast cancer or other) were recorded, as described earlier. The score data were correlated with tumor grade, size, lymph node status, histological subtype, estrogen receptor (ER), progesterone receptor (PR) and Her2. ${ }^{12}$ Two-tailed nonparametric Spearman's correlation test was used. Univariate survival analysis was performed by Kaplan-Meier method; log rank and Breslow tests were applied to estimate differences between the curves. Multivariate analysis was performed using Cox regression proportional hazard models (99\% confidence interval) with a backward stepwise method to remove the least significant variables from the model. The differences were declared significant at $P<0.05$.

\section{Results}

\section{Case Series}

Of 438 invasive breast carcinoma cases included into the tissue microarrays, 258 cases had tumor present in all four tissue cores; this was the only criterion for inclusion of cases in the study. Drop-off of the tissue cores in all other cases was random. The selection of 15 cases of DCIS was random.

Clinicopathological data on the patient series are presented in Table 1. All patients were treated by radical mastectomy or lumpectomy, with or without lymph node dissection, as described earlier. ${ }^{14}$ Adjuvant therapy approaches varied substantially during the period 1974 to 1995 , and no information on individual adjuvant treatment was available.

Table 1 Clinicopathological data on study population (invasive breast carcinoma)

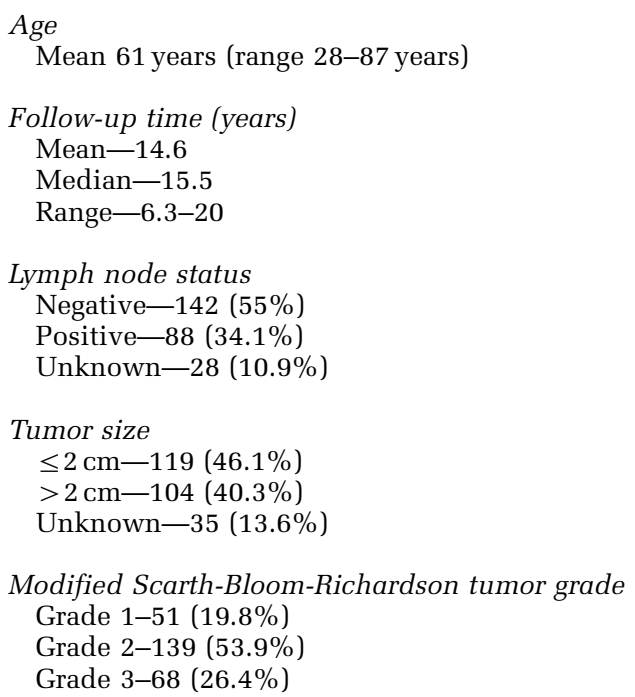


Ethical approval was obtained from the institutional ethical review board to perform this study.

\section{Expression of Stromal CD10 in Breast Cancer and Correlations with other Clinicopathological Data}

There was no strong expression of CD10 in stromal cells of normal breast tissue, but strong CD10 expression was noted in myoepithelial cells of normal breast and faint staining of the specialized stroma of the terminal ductulo-lobular units (Figure 1a). The stromal CD10 immunostaining results in DCIS and invasive breast carcinomas are shown in Table 2. Examples of immunostainings of tissue sections are shown in Figure $1 \mathrm{~b}-\mathrm{d}$. Low grade DCIS showed a trend to negative stromal CD 10
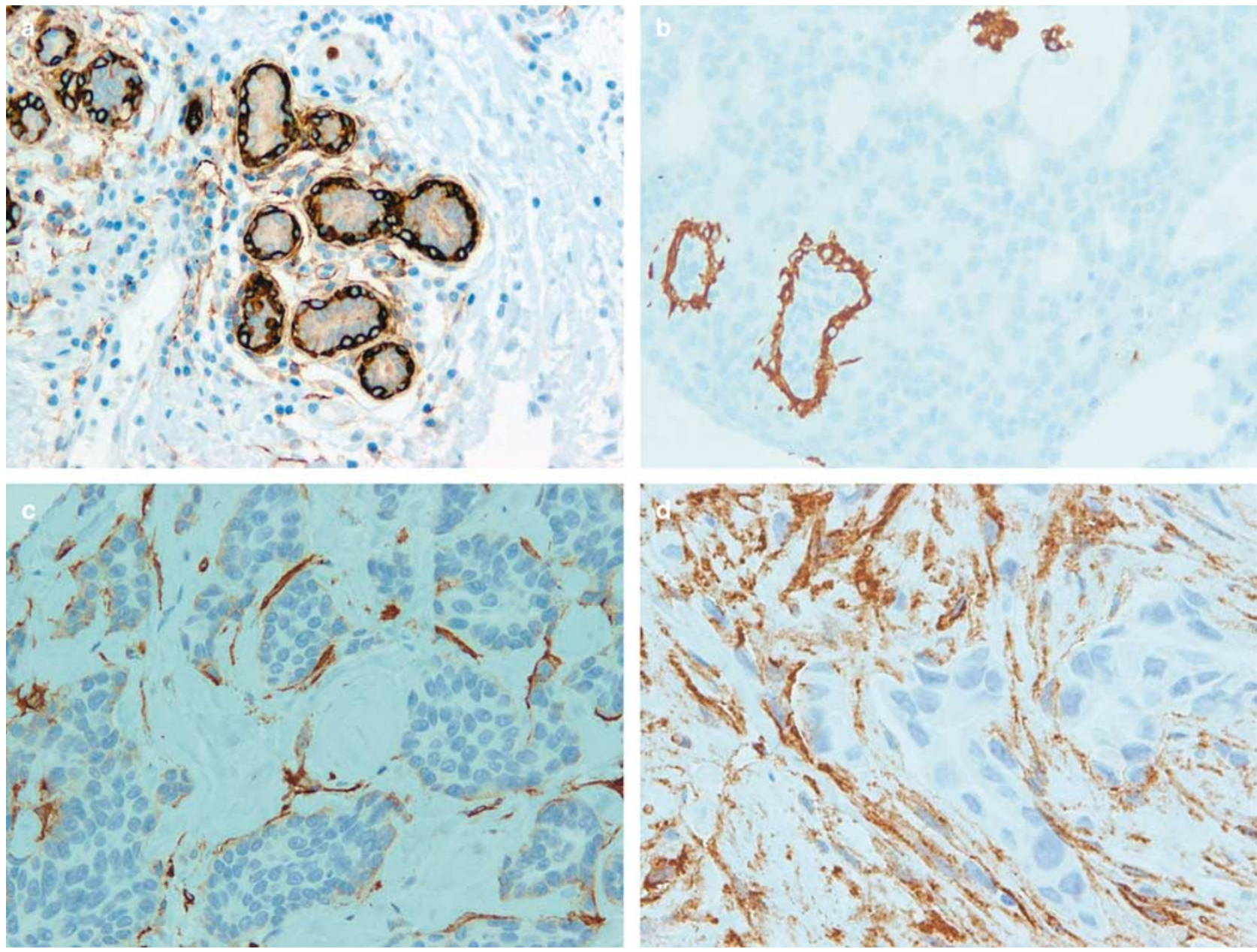

Figure 1 (a) CD10 in normal breast: strong staining in myoepithelial cells. (b) Strong reaction in myoepithelial cells of the pre-existing breast ducts and negative tumor stroma. (c) Weak staining: less than $30 \%$ of stromal cells positive. (d) Strong staining: more than $30 \%$ of stromal cells positive. Immunoperoxidase method, DAB, $\times 200$.

Table 2 Distribution of stromal CD10 immunostaining in DCIS and invasive carcinoma of the breast according to tumor grade

\begin{tabular}{|c|c|c|c|c|}
\hline Stromal CD10 & Low grade & Intermediate grade & High grade & Total \\
\hline \multicolumn{5}{|l|}{ DCIS } \\
\hline Negative & $3 / 3$ & $2 / 7$ & $3 / 5$ & $8 / 15$ \\
\hline Weak & $0 / 3$ & $4 / 7$ & $2 / 5$ & $6 / 15$ \\
\hline Strong & $0 / 3$ & $1 / 7$ & $0 / 5$ & $1 / 15$ \\
\hline \multicolumn{5}{|c|}{ Invasive breast carcinoma } \\
\hline Stromal CD10 & Grade 1 & Grade 2 & Grade 3 & Total \\
\hline Negative & $8 / 51(16 \%)$ & $39 / 139(28 \%)$ & $6 / 68(9 \%)$ & $53 / 258(21 \%)$ \\
\hline Weak & $28 / 51(55 \%)$ & $54 / 139(39 \%)$ & $22 / 68(32 \%)$ & $104 / 258(40 \%)$ \\
\hline Strong & $15 / 51(29 \%)$ & $46 / 139(33 \%)$ & $40 / 68(59 \%)$ & $101 / 258(39 \%)$ \\
\hline
\end{tabular}


staining, as opposed to high grade DCIS (nonsignificant difference, $P=0.1$ ). Stromal CD10 expression in invasive breast carcinoma showed statistically significant positive correlation with higher tumor grade $(P=0.002)$. The overall difference in stromal CD10 expression between DCIS and invasive carcinomas was statistically significant $(P=0.003)$. There was a correlation between stronger stromal CD10 staining and ER-negative status of the tumor $(P=0.002)$. There was no correlation between stromal CD10 expression and lymph node status, tumor size, histological subtype, PR, and Her2 status of the tumor $(P>0.05)$.

\section{Prognostic Significance of Stromal CD10 Expression in Invasive Carcinoma of the Breast and Reproducibility of the Scoring}

As expected, there were no breast-cancer associated deaths in the DCIS group. Only invasive breast carcinoma cases were subjected to survival analysis. Kaplan-Meier survival curves and corresponding $P$-values are shown in Figure 2. Strong positive expression of CD10 in the stroma of invasive breast carcinoma was associated with decreased long-term disease-specific survival and overall survival for the entire cohort (Figure 2a and b). In the lymph node negative subset of patients we observed significant differences in long-term disease-specific survival (Figure 2c) and overall survival (not shown). Paired tests for each stratum were performed (ie CD10 strong vs CD10 negative, CD10 weak vs CD10 negative and CD10 strong vs CD10 weak) and showed significant differences in survival for patients with CD10 positive (either CD10 strong or weak) vs CD10 negative tumor stroma, while there was no difference in either type of survival between CD10 weakly and strongly positive groups. In the lymph node positive group we did not observed statistically significant differences in disease-speci-
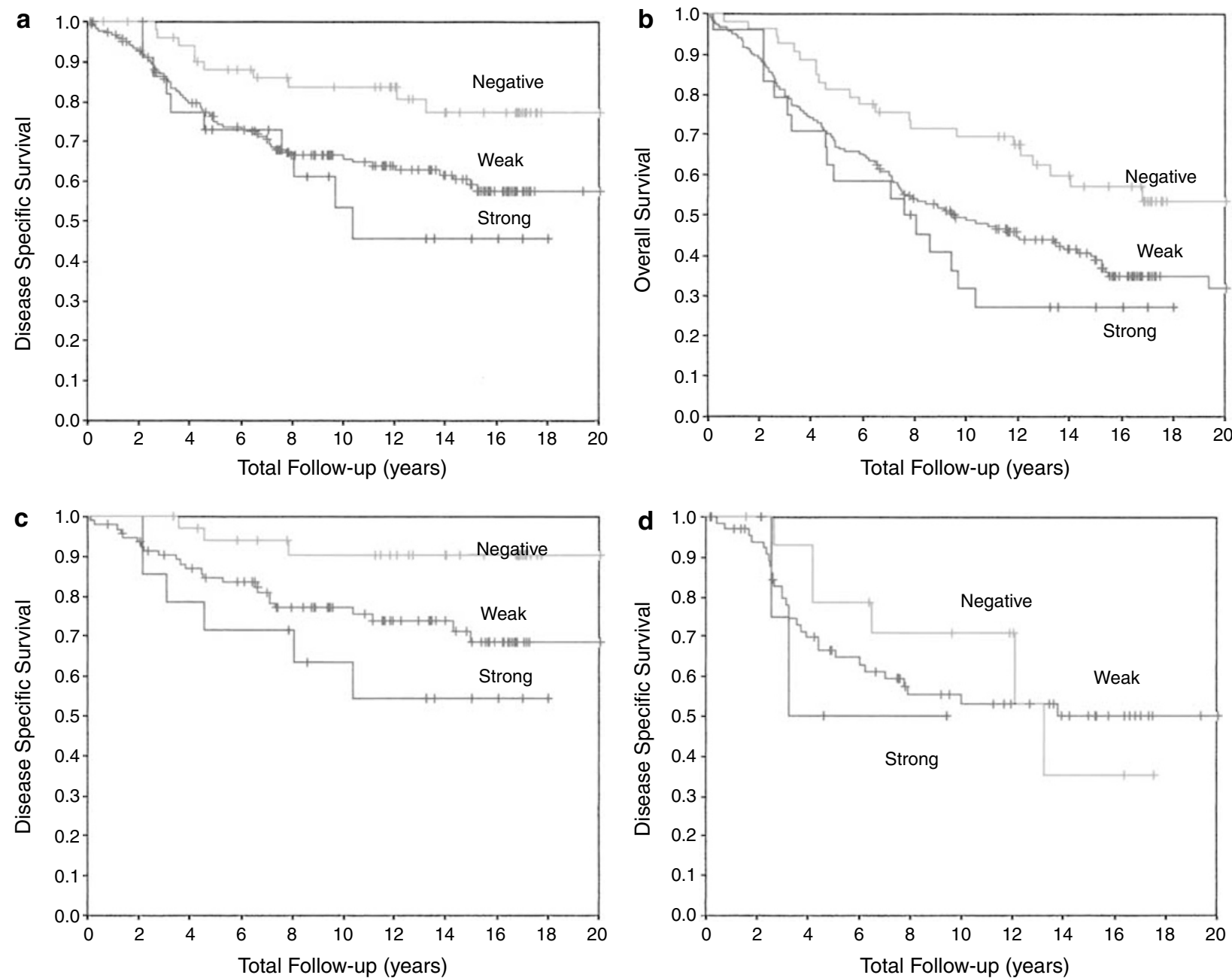

Figure 2 Kaplan-Meier survival analysis of CD10 expression in the stroma of invasive breast carcinoma (log rank/Breslow tests; only one $P$-value is declared in case of no differences between two tests): (a) disease-specific survival, entire cohort: negative $v s$ weak, $P=0.01$; negative vs strong, $P=0.01$; weak vs strong, $P=0.5 / 0.7$. (b) Overall survival, entire cohort: negative vs weak, $P<0.01$; negative vs strong, $P<0.01$; weak vs strong, $P=0.3 / 0.4$. (c) Disease-specific survival, subset analysis, lymph node negative group: negative vs weak, $P=0.03$ / 0.04; negative vs strong, $P<0.01$; weak vs strong, $P=0.3$. (d) Disease-specific survival, subset analysis, lymph node positive group: negative $v s$ weak, negative $v s$ strong, weak vs strong, for all paired tests $P>0.1$. 
fic survival (Figure 2d), while it was a significant difference in overall survival (not shown). Multivariate analysis of disease-specific survival showed that stromal CD10 expression approached significance as an independent negative prognostic factor $(P=0.06)$, when the model included lymph node status, tumor size, ER, and Her2. When the least significant variables were excluded from the model in a stepwise fashion, only lymph node status $(P=0.003)$ and stromal CD10 expression $(P=0.05)$ retained independent prognostic significance. In this model positive stromal CD10 expression was associated with relative risk of death 2.8 compared to relative risk 2.4 produced by lymph node positive status. When overall survival was considered with the same variables in the model, only lymph node status was of independent significance, while CD10 showed a trend towards significance $(P=0.08)$.

Stromal CD10 expression scoring data, provided independently by two pathologists ( $\mathrm{MH}$ and $\mathrm{BC}$ ) on two independent sets of tissue microarrays were subjected to variation analysis. It considered variation in the immunostainings between two sets of double core tissue microarrays and interobserver variation simultaneously. We observed $77 \%$ concordance for score 1 and $65 \%$ for score 2 . Substantial agreement between scoring results was achieved, when the results were binarized as negative and positive $(\kappa=0.6)$, although only fair agreement $(\kappa=0.4)$ was noted when three tier scoring system was used (ie negative, weak and strong). Notably, despite these variations, CD10 retained its prognostic significance, particularly in lymph node negative subset of patients, when survival analysis was performed separately for the sets of scores submitted by two different pathologists (data not shown).

\section{Discussion}

The matrix plays a key role in the development of cancer, as matrix molecules play a modulating role on tumor invasion and metastasis. A better understanding of stromal contributions to cancer progression may identify specific signals that promote growth, de-differentiation, invasion, and ectopic survival, and eventually result in the identification of new therapeutic targets for future treatments. ${ }^{15}$

Matrix metalloproteinases are a family of metallopeptidases that cleave the protein components of extracellular matrix and thereby play a central role in tissue remodeling. Several synthetic matrix metalloproteinases inhibitors have been developed and shown to be effective in blocking tumor growth in experimental animals, validating the concept of matrix metalloproteinases as therapeutic targets for cancer. Unfortunately, synthetic matrix metalloproteinases inhibitors have thus far failed to live up to their expectations in initial clinical trials in humans. ${ }^{16}$
CD10—cell surface zinc-dependent metalloproteinase, has been demonstrated on the stromal cells of some breast carcinomas, and suggested to be upregulated in breast cancer cells. ${ }^{17}$ Some experimental data indicate that CD10 may be a potential target for new cancer therapies, as it is involved in cleavage of doxorubicin, critical component of many cancer treatment protocols, and results in chemoresistance. Inhibition of CD10 enzymatic activity may enhance the antitumor efficacy of traditional chemotherapeutic regimens. ${ }^{18}$

We evaluated overall expression of CD10 in tumor stroma. Indeed, stroma in breast cancer is cellular, and CD10 staining could be observed in both stromal cells and extracellular matrix. It is known that neutral endopeptidase could be found as intracellular as extracellular, since it is produced by specialized tumor stromal cells and, once secreted, is involved in extracellular matrix degradation. To date, only one study has addressed prognostic significance of stromal CD10 expression in invasive carcinoma of the breast. ${ }^{2}$ It was shown that stromal CD10 expression was associated exclusively with invasive breast carcinoma, but not with carcinoma in situ and a higher frequency of stromal CD10 staining was seen primarily with lymph node positive breast cancers. In our study, we confirm the prognostic significance of stromal CD10 expression in invasive breast carcinoma in univariate and multivariate analyses. Stromal CD10 expression was consistently associated with decreased survival in the node-negative group of patients $(P=0.01)$; in node-positive group we observed only a trend towards decreased disease-specific survival $(P>0.1)$. We also found that stromal CD10 expression is not exclusive for invasive carcinomas, as was suggested earlier, ${ }^{2}$ as CD10 stromal positivity was present in $47 \%$ of cases of DCIS.

A recent gene expression profiling study of breast carcinoma stroma ${ }^{19}$ identified two clinically significant types of stromal signatures in breast cancer, namely, solitary fibrous tumor type and desmoidtype fibromatosis type, where the first was associated with poor outcome. These two types of stromal signatures were independent from gene clusters previously described ${ }^{20,21}$ in multivariate analysis, indicating that both tumor-cell-mediated and stromal-cell-mediated pathways are of independent significance for the natural history of breast carcinoma. CD10 expression was associated preferentially with desmoid-type fibromatosis stromal signature, and, possibly, contributed to a number of negative outcomes in invasive carcinoma of the breast with this type of stromal signatures. This may suggest that CD10 constitutes a component of a novel independent stromal signaling pathway, which contributes to biological and clinical aggressiveness of invasive breast carcinoma.

We conclude that expression of CD10 in the stoma of invasive breast carcinoma is associated ERnegativity, higher tumor grade, decreased patient 
survival, most significant in the node-negative subset. CD10 constitutes a clinically important prognostic marker and a potential target for development of novel therapies. Further functional studies are needed to elucidate signaling mechanisms resulting in over-expression of CD10 in the stroma of invasive breast carcinoma.

\section{Acknowledgements}

We are grateful to Maggie Cheang, Dmitry Turbin, and Tao Fan for technical assistance and data handling. This work was supported in part by an educational grant from Sanofi aventis Canada. Dr Dabiri is a visiting Professor of Pathology from the University of Kerman, Iran.

\section{References}

1 Albrecht M, Gillen S, Wilhelm B, et al. Expression, localization and activity of neutral endopeptidase in cultured cells of benign prostatic hyperplasia and prostate cancer. J Urol 2002;168:336-342.

2 Iwaya K, Ogawa H, Izumi M, et al. Stromal expression of CD10 in invasive breast carcinoma: a new predictor of clinical outcome. Virchows Arch 2002;440:589-593.

3 Kesse-Adu R, Shousha S. Myoepithelial markers are expressed in at least $29 \%$ of oestrogen receptor negative invasive breast carcinoma. Mod Pathol 2004;17:646-652.

4 Ogawa H, Iwaya K, Izumi M, et al. Expression of CD10 by stromal cells during colorectal tumor development. Hum Pathol 2002;33:806-811.

5 Tokuhara T, Adachi M, Hashida H, et al. Neutral endopeptidase/CD10 and aminopeptidase N/CD13 gene expression as a prognostic factor in non-small cell lung cancer. Jpn J Thorac Cardiovasc Surg 2001;49:489-496.

6 Kristiansen G, Schluns K, Yongwei Y, et al. CD10 expression in non-small cell lung cancer. Anal Cell Pathol 2002;24:41-46.

7 Popnikolov NK, Ayala AG, Graves K, et al. Benign myoepithelial tumors of the breast have immunophenotypic characteristics similar to metaplastic matrixproducing and spindle cell carcinomas. Am J Clin Pathol 2003;120:161-167.

8 Kalof AN, Tam D, Beatty B, et al. Immunostaining patterns of myoepithelial cells in breast lesions: a comparison of CD10 and smooth muscle myosin heavy chain. J Clin Pathol 2004;57:625-629.

9 Moritani S, Kushima R, Sugihara H, et al. Availability of CD10 immunohistochemistry as a marker of breast myoepithelial cells on paraffin sections. Mod Pathol 2002;15:397-405.

10 Huang WB, Zhou XJ, Chen JY, et al. CD10-positive stromal cells in gastric carcinoma: correlation with invasion and metastasis. Jpn J Clin Oncol 2005;35: 245-250.

11 Tse GM, Tsang AK, Putti TC, et al. Stromal CD10 expression in mammary fibroadenomas and phyllodes tumours. J Clin Pathol 2005;58:185-189.

12 Consensus Conference on the classification of ductal carcinoma in situ. The Consensus Conference Committee. Cancer 1997;80:1798-1802.

13 Liu CL, Prapong W, Natkunam Y, et al. Software tools for high-throughput analysis and archiving of immunohistochemistry staining data obtained with tissue microarrays. Am J Pathol 2002;161:1557-1565.

14 Makretsov NA, Huntsman DG, Nielsen TO, et al. Hierarchical clustering analysis of tissue microarray immunostaining data identifies prognostically significant groups of breast carcinoma. Clin Cancer Res 2004;15:6143-6151.

15 De Wever O, Mareel M. Role of tissue stroma in cancer invasion. J Pathol 2003;200:29-47.

16 Coussens LM, Fingleton B, Matrisian LM. Matrix metalloproteinase inhibitors and cancer: trials and tribulations. Science 2002;295:2387-2392.

17 Atherton AJ, O’Hare MJ, Buluwela L, et al. Ectoenzyme regulation by phenotypically distinct fibroblast subpopulations isolated from the human mammary gland. J Cell Sci 1994;107:2931-2939.

18 Pan C, Cardarelli PM, Nieder MH, et al. CD10 is a key enzyme involved in the activation of tumor-activated peptide prodrug CPI-0004Na and novel analogues: implications for the design of novel peptide prodrugs for the therapy of CD10+ tumors. Cancer Res 2003;63: 5526-5531.

19 West RB, Nuyten DS, Subramanian S, et al. Determination of stromal signatures in breast carcinoma. PLoS Biol 2005;3:1101-1110.

20 van de Vijver MJ, He YD, van't Veer LJ, et al. A geneexpression signature as a predictor of survival in breast cancer. N Engl J Med 2002;347:1999-2009.

21 Sorlie T, Tibshirani R, Parker J, et al. Repeated observation of breast tumor subtypes in independent gene expression data sets. Proc Natl Acad Sci USA 2003;100:8418-8423. 